\title{
New beam-combination techniques at IOTA
}

\section{Wesley A. Traub, Angela Ahearn, Nathaniel P. Carleton, Jean-Philippe Berger, Michael K. Brewer, et al.}

Wesley A. Traub, Angela Ahearn, Nathaniel P. Carleton, Jean-Philippe Berger, Michael K. Brewer, Karl-Heinz Hofmann, Pierre Y. Kern, Marc G. Lacasse, Fabien Malbet, Rafael Millan-Gabet, John D. Monnier, Keiichi Ohnaka, Ettore Pedretti, Sam Ragland, F. Peter Schloerb, Kamal Souccar, Gerd Weigelt, "New beam-combination techniques at IOTA," Proc. SPIE 4838, Interferometry for Optical Astronomy II, (21 February 2003); doi: $10.1117 / 12.459718$

Event: Astronomical Telescopes and Instrumentation, 2002, Waikoloa, Hawai'i, United States 


\title{
New Beam-Combination Techniques at IOTA
}

\author{
W.A. Traub ${ }^{a}$, A. Ahearn ${ }^{a}$, N.P. Carleton ${ }^{a}$, J.-P. Berger ${ }^{a, b}$, M. Brewer ${ }^{c}$, K.H. Hofmann ${ }^{d}$, \\ P. Kern ${ }^{b}$, M.G. Lacasse ${ }^{a}$, F. Malbet ${ }^{b}$, R. Millan-Gabet ${ }^{a, e}$, J.D. Monnier ${ }^{a, f}$, \\ K. Ohnaka ${ }^{d}$, E. Pedretti ${ }^{a, g}$, S. Ragland ${ }^{a}$, F.P. Schloerb ${ }^{c}$, K. Souccar ${ }^{c}$, and G. Weigelt ${ }^{d}$ \\ ${ }^{a}$ Harvard-Smithsonian Center for Astrophysics, 60 Garden St., Cambridge MA, 02138, USA \\ ${ }^{b}$ LAOG, Observatoire de Grenoble, Grenoble, France \\ ${ }^{c}$ University of Massachusetts, Amherst, MA, USA \\ ${ }^{d}$ Max-Planck-Institut fur Radioastronomie, 53121 Bonn, Germany \\ ${ }^{e}$ Interferometry Science Center, Caltech, Pasadena, CA, USA

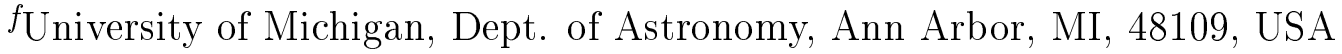 \\ ${ }^{g}$ College de France, Place Marcelin Berthelot, Paris, France
}

\begin{abstract}
New beam combination techniques, using two and three telescopes, have been the focus of activity at IOTA during the past two years since our last update. ${ }^{1}$ In particular, we have added a third telescope, made closure-phase measurements, demonstrated two- and three-beam combination with integrated optics combiners, demonstrated two-beam combination with an asymmetric coupler, and made simultaneous JHK visibility measurements with an image-plane combiner.
\end{abstract}

Keywords: interferometer, beam combination

\section{INTRODUCTION}

IOTA was originally built as a two-telescope interferometer, but it was designed with several features that would allow a relatively easy transition to a three-telescope interferometer. ${ }^{2,3}$ IOTA was also designed to be able to test innovative ideas in interferometry, and to capitalize on these innovations by making observations that would otherwise not be possible. We believe that IOTA has demonstrably lived up to these original expectations. We show here that innovation and new observations are continuing to shape the future at IOTA.

\section{THIRD TELESCOPE ADDITION}

Although IOTA was built on a modest budget, we were nevertheless able to design it so that a future transition from two to three telescopes would be feasible, with only modest changes to the existing structure. In association with our recently-completed third-telescope upgrade, the most significant recent changes are in the areas of (1) telescope location, (2) long delay line, (3) short delay line, (4) star tracker, (5) beam combination, (6) computer control system, (7) reconfigurable electronics, (8) closure-phase measurements, and (9) fringe-packet tracking.

Further author information: W.A.T.: email wtraub@cfa.harvard.edu; tel. 617-495-7406

J.P.B.: Michelson Postdoctoral Fellow when work was done, currently at Grenoble

R.M.G.: Michelson Postdoctoral Fellow when work was done, currently at Caltech

J.D.M.: Smithsonian Postdoctoral Fellow when work was done, currently at U. Michigan

E.P.: Smithsonian Predoctoral Fellow

S.R.: Michelson Postdoctoral Fellow 


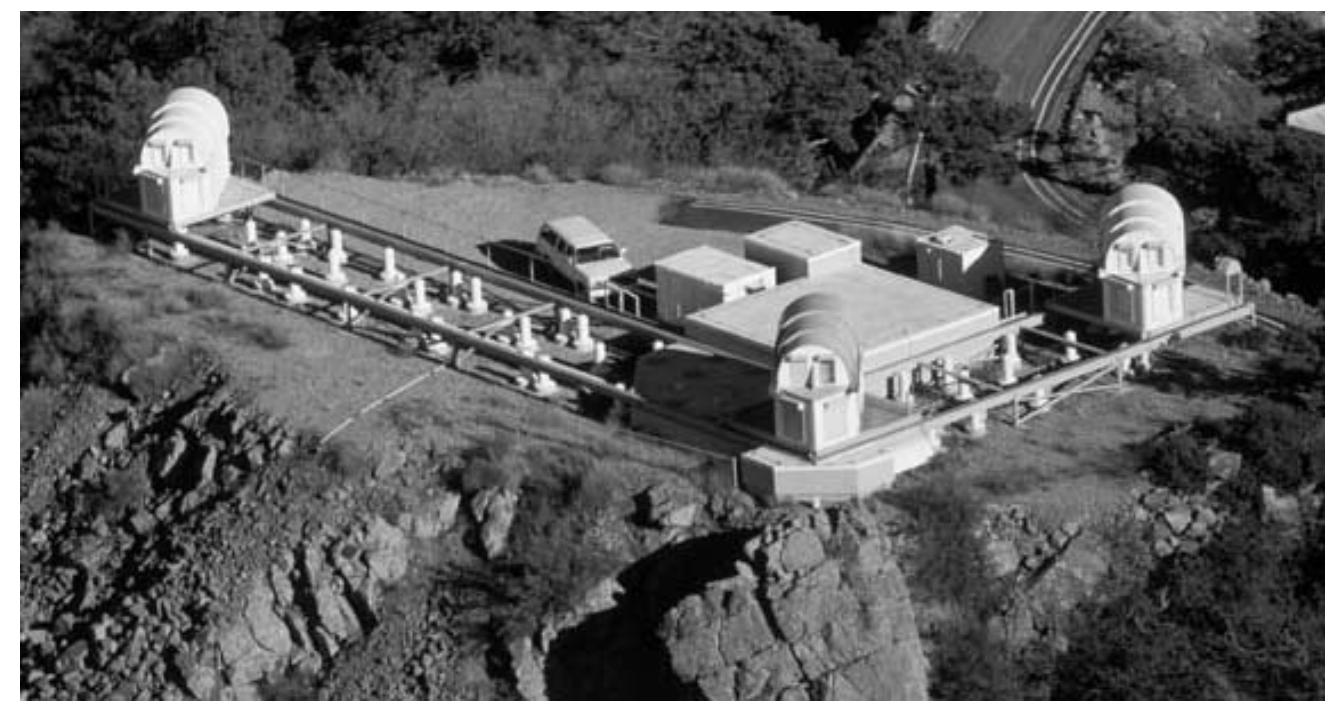

Figure 1. The IOTA interferometer on Mt Hopkins. All 3 telescopes are moveable, but shown here at their extreme end stations. Telescope A is left, telescope B is right, and telescope $\mathrm{C}$ is center. Both A and C move on the long (35 m) arm, and $\mathrm{C}$ moves on the short $(15 \mathrm{~m})$ arm.

\subsection{Telescope Location}

The IOTA telescopes are mounted within transportable shelters that move along I-beam rails. The rails form an "L" with arm lengths of $35 \mathrm{~m}$ toward the north-east (telescope A) and $15 \mathrm{~m}$ toward the south-east (telescope B), from a corner position toward the west (telescope $\mathrm{C}$ ), where the arms meet. The arm lengths are constrained by the geometry of the site, with rather steep elevation gradients just beyond both ends of each arm. All telescopes and shelters are essentially identical. The third telescope (C) can be positioned at any station between the corner and telescope A. Measured from the corner, stations are located at distances that are multiples of 5 or 7 m, i.e., at $0,5,7,10,14,15,20,21,25,28,30$, and $35 \mathrm{~m}$. For the work reported here, telescope $\mathrm{C}$ was located at the corner. A photograph of the interferometer is shown in Fig. 1, where telescope A is at $35 \mathrm{~m}$ on the left, B is at $15 \mathrm{~m}$ on the right, and $\mathrm{C}$ is at $0 \mathrm{~m}$ in the foreground.

\subsection{Long Delay Line}

There are two long vacuum delay lines, LD1 and LD2, for the three telescopes. Telescopes A or B can be switched to feed either LD1 or a fixed (non-moveable) set of mirrors. The switching is carried out by moving a pair of corner mirrors, depending upon which telescope requires the largest delay, for a given target star. Telescope $\mathrm{C}$ is permanently assigned to LD2. Both LD1 and 2 comprise dihedral mirrors mounted on sliding carts that sit on flat pieces of steel bar stock rails, and the carts are pulled by loops of steel wire driven by stepper motors. The rails are mounted from the bottom flange of a steel I-beam, so that the carts ride between the top and bottom flanges. The whole assembly is contained in a close-fitting circular cylindrical steel pipe with vacuum joints made up of the outer cylindrical rubber bands that were pioneered at IOTA. The location of each cart is measured with an HP laser interferometer operating at $0.633 \mathrm{~nm}$ wavelength, with the laser located in air, just beyond the 35-m station. To accommodate telescope $\mathrm{C}$, we merely had to install the LD2 cart and laser-based control mechanisms.

\subsection{Short Delay Line}

The short delay line is a granite surface equipped with a single air-bearing track and motor on its top surface, located near the corner. It is in the same vacuum chamber as the long delay lines, and is bore-sighted with them. To accommodate the third telescope we added a second air-bearing cart (SD2), in series with the first one (SD1), both on the same linear motor track, but driven by independently activated currents in coils mounted 
on each cart. Cart SD1 is assigned to either telescope A or B, and SD2 is dedicated to telescope C. Although the carts are in series, the dihedral mirror unit mounted on each is offset transversely, so that both beams can be delayed in parallel. The presence of SD2 means that the range of travel of SD1 is somewhat shorter than it was originally; the range of each cart is about $2.1 \mathrm{~m}$ of physical motion. Each is controlled by a second HP laser interferometer, with the laser light originating near the corner position. It is a curious and useful property of IOTA that, to track a star, the SD1 and SD2 carts will always move away from each other; thus, if the carts are correctly positioned at a given time, they will not collide at a later time while tracking that star, while it is in range of the telescopes.

\subsection{Star Tracker}

IOTA originally had two separate star-tracker CCDs, one per telescope. However to accommodate the third telescope without building a third such unit, we decided to reconfigure the optics so that all three beams now fall on a single CCD, one of the original pair. Each star image is directed to its own quadrant of the CCD. To allow the star images to be uniquely identified during the star-acquisition process, we added a normally-open shutter to each beam. The field of view of each quadrant is about 16 arcsec on a side, which is about the same as the unvignetted field of view from each telescope, so these fields are approximately matched; if a star from one quadrant finds itself in a neighboring quadrant it will be noticeably attenuated. In the coming year we expect to replace this CCD chip with one with a larger quantum efficiency, a faster set of electronics, and lower read noise.

\subsection{Beam Combination}

Until recently, IOTA enjoyed the use of (a) a classical multimode two-beam combination system using a freespace "half-silvered" combiner plate and a NICMOS3 detector, ${ }^{4}$ and (b) the single-mode two-beam fiber-optic combiner FLUOR ${ }^{5}$ and the same NICMOS3 detector. To prepare for three-beam operation, the first system has been dismantled, and the NICMOS3 detector replaced by a PICNIC detector. ${ }^{6}$ The FLUOR system was moved to the CHARA interferometer in July 2002. On a short-term basis, IOTA benefited from a brief but productive visit in 2001 of the JHK spectrophotometric image-plane combiner from MPI in Heidelberg, ${ }^{7}$ which will be discussed below. Subsequently we have installed three other combiners, a free-space classical three-beam combiner, a single-mode two- and three-beam set of integrated optics combners, ${ }^{8}$ and a single-mode two-beam visible combiner. ${ }^{9}$ Each of these is discussed below.

\subsection{Computer Control System}

IOTA's original Macintosh computers turned out to be inadequate to control the significantly more complex three-telescope operation. Their communication speed and computing rates were both too limited. The system suffered from the inability of computers controlling various subsystems to communicate, forcing the observer to re-enter the same data into different computers for each observation.

The replacement system is based on three Motorola MPC750 Power PC single board computers mounted in a 21 slot VME backplane. The first computer runs the telescope tracking software and controls a commercial eight-axis stepper motor controller, replacing the in-house designed stepper rate cards used with the Macintosh system. Six of the eight axes are used for controlling the roll and tilt axes of the three telescopes and the other two axes control the short delay lines. The second computer is dedicated to running the star tracker. It uses a CPLD digital I/O card programmed to read the star tracker CCD and controls a 6 channel current loop D/A card operating the star tracking tip-tilt mirrors on all three telescopes. The third computer is used for data aquisition. It uses a second CPLD digital I/O card programmed to read the camera and controls $2 \mathrm{D} / \mathrm{A}$ channels running the scanning piezo mirrors. This computer also runs the fringe tracking software and generates offsets for the short delay lines. All three computers run the VxWorks real time operating system and communicate with each other using dedicated blocks of shared memory on each card. All of the VxWorks software is written in object oriented $\mathrm{C}++$.

The VxWorks host computer is a Sun Blade 1000 workstation running the Solaris- 8 operating system. This computer contains a fiber optic PCI to VME bridge which gives it access to the shared memory on the VME single board computers. The graphical user interface, written in Java, runs on the Sun host. All control and 


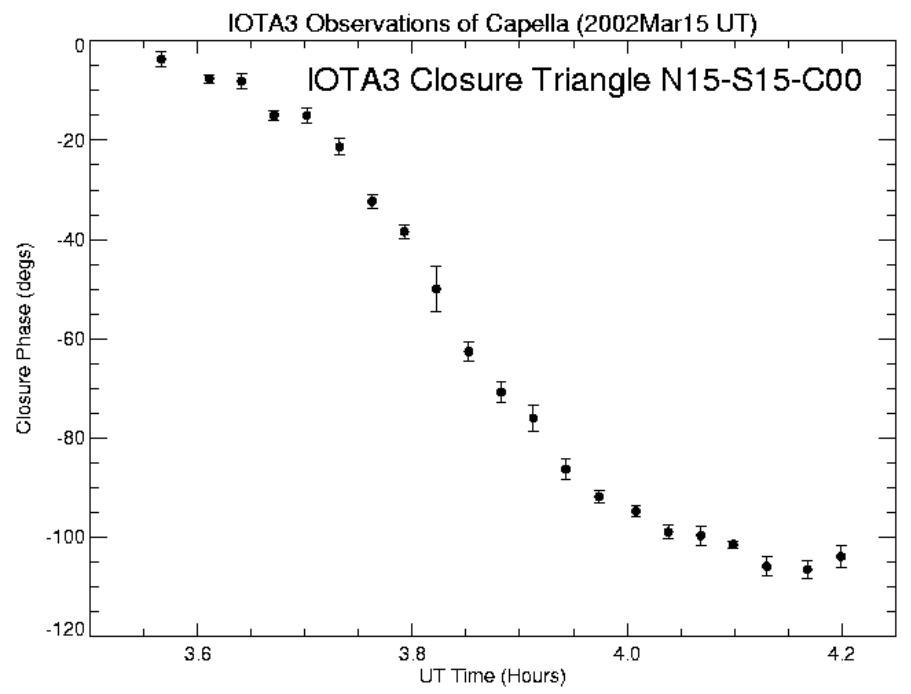

Figure 2. Closure-phase observed on Capella with the IONIC integrated-optics 3-beam combiner in early 2002, soon after initial operation of all three telescopes simultaneously.

monitoring functions are implemented through data exchange via shared memory. The user interface contains a scripting tool which allows the observer to save and restore custom telescope setup and observation procedures. The new IOTA monitor and control system is based on that of the Large Millimeter Telescope project being developed by the University of Massachusetts, for which IOTA is serving as a test site.

\subsection{Reconfigurable Electronics}

As part of the new control system at IOTA, the clocking and readout of our PICNIC science detector and CCD star-tracker detector is now controlled by a complex programmable logic device (CPLD), rather than by dedicated hardware or by a computer. We use one Altera CPLD for the PICNIC chip, and another for our star-tracker CCD. Each CPLD is mounted close to the single-board computer with which it is associated, and through which the acquired data flows. Pedretti et al. ${ }^{6}$ give a full description of these applications at IOTA.

There are several advantages to using a CPLD for detector readout. For us, the main advantages are that several readout mode programs can be stored and quickly downloaded as needed, and that we can easily change the program details if needed. Some disadvantages that we have found are that the actual timing of the clocking signals can vary from what is expected, that the learning curve is rather long, and that the close proximity of mounting near the single-board computer chip can cause overheating which in our case will produce detector output spikes if the room temperature is allowed to climb above about $71^{\circ} \mathrm{F}$. Nevertheless these are useful devices, and we are pleased with their performance.

\subsection{Closure-phase Measurements}

The concept of closure phase in optical-infrared interferometry is well known. ${ }^{10}$ With three telescopes at IOTA, we have several advantages over just two telescopes. One advantage is simply that we could operate pairwise and enjoy the tripled number of baselines. Another advantage is that in closure-phase mode we can servo the delays to make the two shorter baseline fringes appear at about the same time, and thereby automatically force the third baseline fringes to be present, which is useful if the object is resolved on that baseline so that the fringes are weak (baseline bootstrapping). The third advantage is the main one however, giving us the quantity of closure phase, which is a measure of the degree of point-reflection asymmetry in the source.

Our third telescope became fully operational on 23 February 2002, and we achieved closure-phase operation a few weeks later on 14 March 2002, on Capella. An example is shown in Fig. 2. 


\subsection{Fringe-packet tracking}

A crucial requirement for closure-phase measurements is that the six fringe packets (or three pairs) be measured approximately simultaneously in time. If this requirement is not met, then we might be measuring fringes which have passed through significantly different optical paths, due to air turbulence, and the closure-phase would be meaningless. Accordingly, we have worked to develop a fringe-packet follower, the output of which can be fed to an appropriate delay mirror for compensation. We have successfully implemented several versions of such an algorithm, and have used one of these regularly to make our three-beam measurements. A description of the algorithms and their performance is being developed by Ettore Pedretti, as part of his $\mathrm{PhD}$ thesis work, and will be published in the coming year.

\section{INTEGRATED-OPTICS IONIC COMBINERS}

The IONIC integrated-optics two-beam combiner at IOTA $^{11}$ was the first integrated optics device to make an astronomical measurement. When IOTA added its third telescope, an upgraded version, the three-beam IONIC combiner was already in place. Fringe modulation is controlled by 60 -micron stroke piezo-driven infraredreflecting dichroics in the path of two of the three combining beams, with a triangle ramp drive speed in one that is twice that of the other. The resulting time-modulated fringe outputs on two baselines are therefore at a single temporal frequency, and the fringes on the remaining baseline are at twice that frequency. The first results were observations of Capella, for which a sample phase-closure plot is shown in Fig. 2. A full description of the three-beam combiner is given in this volume by Berger et al.. ${ }^{8}$

Among the advantages of the present 3-beam IONIC combiner are: automatic single-mode spatial filtering at the input feed fiber; ability to recover intensity fluctuations without the need for separate photometric taps on the beams; invariance of closure phase to room temperature (at least in experience to date); ease of focussing of output beams onto our PICNIC detector array; convenience of being able to back-illuminate the fibers so as to produce a beam which can be matched to the star tracker and to the feed telescopes, thereby simplifying each night's initial alignment.

There are a few disadvantages to our present setup, chief of which are: the need for an output polarization analyzer that cuts the light intensity by more than a factor of two; and the apparent polarization sensitivity of the feed fibers to changes of mechanical orientation. To overcome these, we initially align the fibers with a temporary input polarizer, and rotate the fiber until the output intensity, as measured through a second polarizer at the output, is maximized. We also try to ensure that the fibers are not mechanically disturbed, once this polarization orientation has been set.

The issues of improving the polarization sensitivity, and maximizing the intrinsic throughput of IONIC, are topics of ongoing research. Present plans call for the installation of an improved IONIC in November 2002.

\section{ASYMMETRIC COMBINER}

For ground-based interferometers, it is often said of single-mode spatial filters that these devices essentially trade visibility fluctuations for intensity fluctuations. In other words, without a spatial filter essentially all the transmitted light from each telescope reaches the detector, but since the wavefront is constantly being perturbed by the atmosphere, the degree of coherence will fluctuate, and the fringe amplitude will vary quite a bit. On the other hand, if the star is focussed onto a single-mode fiber either before or after combination, then only the plane wave part of the wavefront is transmitted and the resulting fringe visibility is constantly maximized, however since the star image will frequently be so distorted by the atmosphere that part will fall outside the fiber, there will be strong variations of the intensity of transmitted light. In some fiber systems, this problem is solved by tapping off a portion of the light after it enters the fiber, and before combination. This tapped beam can then be detected in a separate photometric channel, and used to scale the instantaneous fluctuations of the combined beams.

A disadvantage of this scheme is that the tap removes a fraction of the collected light, which reduces the available signal, and also the photometric beam requires a separate detector channel, which reduces the time available for measuring the interferred signal. 
A scheme which goes partway to solving this problem was suggested by Monnier, ${ }^{12}$ and implemented at IOTA recently. ${ }^{9}$ The idea is to build an asymmetric coupler, such that the light is not split 50:50, but rather in a different proportion, say about 70:30. This does not affect the degree of interference too much, because the interference is between amplitudes and not intensities. However it does provide some information at each combined output regarding the relative input intensities to the two fibers which make up the combiner, since the degeneracy of inputs at the output is now lifted. A two-beam version of this device was recently successfully demonstrated at IOTA at visible wavelengths. The first application will be to measure Cepheid diameters at about 0.8 micron wavelength.

\section{FREE-SPACE COMBINER}

We recently installed at IOTA a three-beam free-space combiner, using conventional bulk optics beamsplitters and combiners, but equipped at the outputs with fibers which can be either single-mode or multi-mode. This combiner uses Infrasil 301 plates which are coated to be approximately $50 \%$ reflecting and $50 \%$ transmitting over the full range from visible $(0.5 \mu \mathrm{m})$ to near-infrared $(2.5 \mu \mathrm{m})$. The three beams are combined pair-wise at three combiner plates, all mounted on a common piezo-driven platform, so that the output fringe frequencies for all three baselines are at the same frequency.

The output beams are focussed onto six fibers, the other ends of which are mounted close together in a common multi-groove V-block, so that the image of all six fibers can be focussed onto our PICNIC detector, using the same optics as for the IONIC combiner. The free-space combiner has not yet been fully aligned and tested. Our initial efforts have gone into the integrated optics combiner instead, but we intend to bring this combiner on-line in the near future. We anticipate that it will be instructive to compare the performance of these combiners side-by-side. One feature of the free-space combiner is that it should be polarization-free. On the other hand, the distance between the points where we split and combine the beams is on the order of a meter, so temperature changes in the lab are likely to be a much more important factor for the free-space combiner than for the integrated-optics combiner.

\section{MAX-PLANCK-INSTITUT JHK-BEAM COMBINER}

In June 2001 Gerd Weigelt and his group brought their two-beam combiner to IOTA for a very productive observing run. ${ }^{7,13,14}$ This image-plane combiner disperses fringes across the full JHK (1.0 to $2.5 \mu \mathrm{m}$ ) range simultaneously, and reads out the two-dimensional dispersed spectrum via a Hawaii detector chip. The image-plane beam-combination method used in this system is an excellent counter-example to the pupil-plane combination method used in all other combiners to date at IOTA. For details, see the referenced papers.

\section{SAO JHK-SPECTROGRAPH}

A proposed future capability at IOTA is the low-resolution spectrograph that is described in this volume by Ragland. ${ }^{15}$ The scientific goal of this spectrograph is to allow us to observe in spectral bands which are narrower than the usual JHK bands, but by only a modest factor of about 3. We chose this resolution as a good match to Mira stars, that in many cases show rather strong line absorption at the edges of the JHK bands. In order to see the continuum, without strong contamination from these blanketing lines, it is necessary to select about the central $1 / 3$ of each band. For comparison, it would be useful to observe as well in the absorption wings, as a separate measurement. Thus we have designed a prism spectrograph which gives nearly constant dispersion across the full JHK range, and will allow these spectra to be displayed simultaneously, for all six output beams from the three telescopes, on our current PICNIC detector chip. Until this device is built, we have installed equivalent narrow-band filters, in the double filter-wheel assembly inside the PICNIC dewar, and will be using these filters to observe Miras and other objects in the near future. 


\section{APERTURE MASKING AND LONG-BASELINE INTERFEROMETRY}

The combined power of aperture masking at a large telescope plus a long-baseline interferometer gives the advantage of being able to cover a large range of baselines. We have exploited this combination using the Keck aperture masking apparatus and the IOTA interferometer, as described in this volume by Monnier et al. ${ }^{16}$ and Millan-Gabet et al.. ${ }^{17}$ For two of the examples shown by Monnier et al., the visibility curves have strong variations from short to long baselines, owing to the source structure of an extended and irregular dust shell, with a more point-like star at its center. The Keck aperture masking at baselines up to $10 \mathrm{~m}$ measures the extended structure in some detail. The long-baseline IOTA measurements establish the high spatial frequency content coming primarily from the stellar core. Together these results allow a complete separation of the signals from each component.

\section{RECENT TWO-TELESCOPE RESULTS}

Prior to the installation of the full three-telescope system, IOTA continued its work with the original two telescopes. In this volume Millan-Gabet et al. ${ }^{17}$ report several of the recent results. One area is multi-color measurements of Mira stars, where we find that the average Mira has an effective diameter which increases slowly with wavelength, such that in going from $\mathrm{J}$ to $\mathrm{H}$ the diameter increases by about $5 \%$, and in going from $\mathrm{H}$ to $\mathrm{K}$ the diameter increases by about $14 \%$.

Another result reported there is the measurement of the x-ray binary CI Cam, where we show that the dust shell has essentially constant diameter over a large range of postion angles, in both the $\mathrm{H}$ and $\mathrm{K}$ bands. However model fitting on the basis of spectral energy distribution data yields a dust shell diameter which is much larger than indicated by our visibility data, by a large factor.

Additional 2-telescope results are the Keck-IOTA observations mentioned above. ${ }^{16}$

Earlier Mira observations at IOTA in the $\mathrm{K}$ and $\mathrm{L}$ bands were recently published by Mennesson et al. ${ }^{18}$ and Chagnon et al. ${ }^{19}$ showing surprisingly large extended atmospheres in Miras at L-band compared to K-band.

Other significant IOTA work published recently includes the measurement at $\mathrm{K}$ band of the diameter of the Cepheid zeta Gem by Kervella et al.. ${ }^{20}$

Also Hoffmann et al. ${ }^{21}$ published the results of diameter measurements of several Miras using the FLUOR 2-beam combiner at IOTA, including a comparison with numerical model atmospheres.

Millan-Gabet et al. ${ }^{22}$ published their observations of Herbig Ae/Be stars, showing that the dust shells around these stars exhibit evidence for rotational symmetry, and more important that the dust shells had unexpectedly large clear areas close to the star. These results have stimulated new models of the dust shells around Herbig Ae/Be stars.

\section{ACKNOWLEDGMENTS}

We are grateful to the the Smithsonian Institution for its ongoing support of infrastructure and research at

IOTA. We acknowledge NASA for its support of the third telescope addition to IOTA. We also acknowledge the NSF for its support of instrumentation and personnel at IOTA.

\section{REFERENCES}

1. W. A. Traub and et al., "The third telescope project at the IOTA interferometer," Proc. SPIE 4006, 2000.

2. N. P. Carleton, W. A. Traub, M. G. Lacasse, P. Nisenson, M. R. Pearlman, R. D. Reasenberg, X. Xu, C. Coldwell, A. Panasyuk, J. A. Benson, C. Papaliolios, R. Predmore, F. P. Schloerb, H. M. Dyck, and D. Gibson, "Current status of the IOTA interferometer," Proc. SPIE 2200, 1994.

3. W. A. Traub and et al., "Recent results from the IOTA interferometer," Proc. SPIE 3350, 1998.

4. R. Millan-Gabet, F. P. Schloerb, W. A. Traub, and N. P. Carleton, "A NICMOS3 camera for fringe detection at the IOTA interferometer," PASP 111, pp. 238-245, 1999.

5. V. C. du Foresto, S. Ridgway, and J. M. Mariotti, "Deriving object visibilities from interferograms obtained with a fiber stellar interferometer," A\&SA 121, pp. 379-392, 1997. 
6. E. Pedretti, R. Millan-Gabet, J. D. Monnier, W. A. Traub, N. P. Carleton, S. Ragland, and M. G. Lacasse, "Reconfigurable electronics at the IOTA interferometer," Proc. SPIE 4838, 2002.

7. C. Weigelt, A. Ahearn, U. Beckman, J. P. Berger, T. Bloecker, M. K. Brewer, Y. N. Efimov, K. Hofmann, M. G. Lacasse, V. Malanushenko, R. Millan-Gabet, J. D. Monnier, K. Ohnaka, D. Schertl, F. P. Schloerb, V. Shenavrin, W. A. Traub, and B. Yudin, "JHK-band spectro-photometry of T Cep with the IOTA interferometer," Proc. SPIE 4838, 2002.

8. J. P. Berger, P. Haguenauer, P. V. Kern, F. Malbet, K. Perraut-Rousselet, I. Schanen-Duport, M. Severi, R. Millan-Gabet, J. D. Monnier, and W. A. Traub, "First results from the IONIC three-beam combiner at IOTA," Proc. SPIE 4838, 2002.

9. J. D. Monnier, R. Millan-Gabet, W. A. Traub, N. P. Carleton, E. Pedretti, C. M. Coldwell, and C. D. Papaliolios, "SMART precision interferometry at 780 nm," Proc. SPIE 4838, 2002.

10. J. D. Monnier, "An introduction to closure phases," in Principles of Long Baseline Stellar Interferometry, P. R. Lawson, ed., pp. 203-230, JPL, 2001.

11. J. P. Berger, P. Haguenauer, P. Kern, K. Perraut, F. Malbet, I. Schanen, M. Severi, R. Millan-Gabet, and W. Traub, "Integrated optics for astronomical interferometry," $A \xi A$ 376, pp. L31-L34, 2001.

12. J. D. Monnier, "Asymmetric beam combination for optical interferometry," PASP 113, pp. 639-645, 2001.

13. K. Hofmann, A. Ahearn, U. Beckman, J. P. Berger, T. Bloecker, M. K. Brewer, Y. N. Efimov, M. G. Lacasse, V. Malanushenko, R. Millan-Gabet, J. D. Monnier, K. Ohnaka, D. Schertl, F. P. Schloerb, V. Shenavrin, W. A. Traub, G. Weigelt, and B. Yudin, "Near-infrared IOTA interferometry of the symbiotic star CH Cyg," Proc. SPIE 4838, 2002.

14. K. Ohnaka, A. Ahearn, U. Beckman, J. P. Berger, T. Bloecker, M. K. Brewer, Y. N. Efimov, K. Hofmann, M. G. Lacasse, V. Malanushenko, R. Millan-Gabet, J. D. Monnier, D. Schertl, F. P. Schloerb, V. Shenavrin, W. A. Traub, G. Weigelt, and B. Yudin, "IOTA observation of the circumstellar envelope of R CrB," Proc. SPIE 4838, 2002.

15. S. Ragland, W. A. Traub, and R. Millan-Gabet, "Low-resolution spectrograph for the IOTA interferometer," Proc. SPIE 4838, 2002.

16. J. D. Monnier, R. Millan-Gabet, P. G. Tuthill, W. A. Traub, N. P. Carleton, V. C. du Foresto, W. C. Danchi, M. G. Lacasse, S. Morel, G. Perrin, and I. L. Porro, "Aperture synthesis using multiple facilities: Keck aperture masking and the IOTA interferometer," Proc. SPIE 4838, 2002.

17. R. Millan-Gabet, E. Pedretti, J. D. Monnier, S. Ragland, W. A. Traub, F. P. Schloerb, N. P. Carleton, and M. G. Lacasse, "Science results from the two-telescope IOTA," Proc. SPIE 4838, 2002.

18. B. Mennesson, G. Perrin, C. Chagnon, V. du Foresto, S. Ridgway, A. Merand, P. Salome, P. Borde, W. Cotton, S. Morel, P. Kervella, W. Traub, and M. Lacasse, "Evidence for very extended gaseous layers around O-rich Mira variables and M giants," ApJ 579, pp. 446-454, 2002.

19. G. Chagnon, B. Mennesson, G. Perrin, V. C. du Foresto, P. Salome, P. Borde, M. Lacasse, and W. Traub, "L'-band interferometric observations of evolved stars," AJ 124, pp. 2821-2832, 2002.

20. P. Kervella, V. C. du Foresto, G. Perrin, M. Scholler, W. A. Traub, and M. G. Lacasse, "The angular diameter and distance of the Cepheid zeta Geminorum," A\&A 367, pp. 876-883, 2001.

21. K. H. Hofmann, U. Beckmann, T. Blocker, D. Schertl, G. Weigelt, M. Wittkowski, V. C. du Foresto, C. Ruilier, M. Lacasse, S. Morel, B. Pras, W. Traub, M. Scholz, V. Shenavrin, and B. Yudin, "Observations of Mira stars with the IOTA/FLUOR interferometer and comparison with Mira star models," New Astronomy 7, pp. 9-20, 2002.

22. R. Millan-Gabet, F. P. Schloerb, and W. A. Traub, "Spatially resolved circumstellar structure of Herbig Ae/Be stars in the near-infrared," ApJ 546, pp. 358-381, 2001. 